Contributions to Game Theory and Management, XIV, 216-226

\title{
Nash Bargaining Solution as Negotiation Concept for Resource Allocation Problem with Groves-Ledyard Mechanism*
}

\author{
Nikolay A. Korgin and Vsevolod O. Korepanov \\ V.A.Trapeznikov Institute of Control Sciences of Russian Academy of Sciences \\ Profsoyuznaya st. 65, Moscow, 117997, Russian Federation \\ E-mail: nkorgin@ipu.ru, vkorepanov@ipu.ru
}

\begin{abstract}
Motivated by research works on Zeuthen-Hicks bargaining, which leads to the Nash bargaining solution, we analyze experimental data of resource allocation gaming with Groves-Ledyard mechanism. The games were designed in the form of negotiation to allow players to reach consensus. Behavior models based on best response, constant behavior, and Nash bargaining solution are defined. Analysis conducted over decisions made by participants shows that a significant share of all decisions leads to an increase of the Nash bargaining value. It is even higher than the share of decisions that are in agreement with the best-response concept. Consensusended games show light attraction to the Nash bargaining solution, it's less than we obtained in games with the mechanism of Yang-Hajek from another class of so-called proportional allocation mechanisms. We discuss differences of consensus-ended games from timeout-ended games, what decisions lead to the situations with the Nash bargaining value increasing and differences between balanced mechanism Groves-Ledyard and unbalanced mechanism Yang-Hajek.
\end{abstract}

Keywords: resource allocation mechanisms, Nash implementation, Nash bargaining solution, Groves-Ledyard mechanism.

\section{Introduction}

Let's consider a player's behavior in an economic situation from two sides:

- goal - what a player strives to increase. For example: goal function at the end of the game or at each step of the game; total or mean or discounted sum of goal function values at each game step ...

- behavior - how a player behaves in order to reach his goal. Examples: best response, learning, strategy thinking, etc.

The research works on Zeuthen-Hicks bargaining, which leads to the Nash bargaining solution (Harsanyi, 1956), (Vetschera, 2018) shows that the Nash function value can be the goal of players' behavior and/or help players achieve consensus in negotiations. We try to analyze the data of players' behavior through this point of view. Paper (Korgin and Korepanov, 2020) stated that this approach gives some insights which motivate us to do similar tests on the data of games with another mechanism.

For our research we use data of the experimental gaming comparison of resource allocation rules described in (Korgin and Korepanov, 2017). In that research,

${ }^{*}$ This work is partially supported by the RFBR, project no. 19-29-07525

https://doi.org/10.21638/11701/spbu31.2021.16 
several mechanisms were compared in setting with transferable and quasi-linear utilities: a mechanism ( $\mathrm{YH}$ ) from the class of proportional allocation mechanisms (Yang and Hajek, 2005, Başar and Maheswaran, 2003), a mechanism (GL) with balanced payments using the Groves-Ledyard rule (Groves and Ledyard, 1977) that gives the efficient solution of the problem as a Nash equilibrium in the players' game introduced in (Korgin, 2016), as well as its modification reducing the dimension of the action space of the players (Korgin, 2016) and a mechanism based on the distributed optimization algorithm problem (Boyd et al., 2011).

In this paper, we analyze decisions made by participants in games with the GL mechanism. Unlike to the research of YH mechanism (Korgin and Korepanov, 2020), GL is a mechanism with balanced transfers and players have no need and possibility to decrease total transfers. Nevertheless, we show that a significant share of all decisions leads to an increase of the Nash bargaining value, and it is even higher than the share of decisions that are in agreement with the best-response concept. We also discuss how these decisions correspond with other types of behavior actively exhibited by participants of these experiments - so-called Constant or Inertia behavior and with the end of the negotiation process in games.

\section{Resource allocation problem}

An organizational system consists of a single Principal and a set $N=\{1, \ldots, n\}$ of players. Principal disposes of some infinitely divisible good in a limited amount $R \in \mathbb{R}^{1}$ and allots it among the players in any proportion.

The utility of each player $i \in N$ in terms of the good $x_{i} \in[0, R]$ allotted to him is described by a function $u_{i}(\bullet): \mathbb{R}^{1} \rightarrow \mathbb{R}^{1}$ belonging to a certain set $U_{i}$ of admissible utility functions.

The set of admissible allocations is

$$
A=\left\{x=\left(x_{1}, \ldots, x_{n}\right): \sum_{i \in N} x_{i} \leq R, x \in \mathbb{R}_{+}^{n}\right\}
$$

the set of possible utility profiles is

$$
U=\left\{u=\left(u_{1}(\bullet), \ldots, u_{n}(\bullet)\right): u_{i}(\bullet) \in U_{i}, i \in N\right\}
$$

The problem is to find an efficient allocation mapping $g(\bullet): U \rightarrow A$ that should maximize the total utility of all players for any utility profile $u \in U$ :

$$
g(u) \in \underset{x \in A}{\operatorname{Arg} \max } \sum_{i \in N} u_{i}\left(x_{i}\right) .
$$

\section{Model}

We consider the model $N=\{1,2,3\}$ with utility functions $u_{i}\left(x_{i}\right)=\sqrt{r_{i}+x_{i}}$, where $r=(1,9,25)$ - profile of "initial endowment" of players or, generally, types of utility functions. These types are given to players and they don't change through a game. Type $r_{i}$ of a player $i$ is private information and generally not known to the Principal and other players. The amount of disposable resource is $R=115$.

An efficient allocation accordingly to the right part of (3) is allocation when the sum of utilities attains a maximum value. For our model, efficient allocation and 
profit of each player:

$$
\begin{aligned}
& x^{\text {eff }}=(49,41,25) \\
& u^{\text {eff }}=u_{1}\left(x_{1}^{\text {eff }}\right)=u_{2}\left(x_{2}^{\text {eff }}\right)=u_{3}\left(x_{3}^{\text {eff }}\right) \approx 7.07
\end{aligned}
$$

\subsection{Incentive incompatibility}

In the case of arbitrary types $r$ the efficient allocation is determined according to:

$$
x_{i}=\left(R+\sum_{i \in N} r_{i}\right) / n-r_{i}, i \in N .
$$

Obviously, being answered about $r_{i}$ any player strives for underrating the value of his parameter (to increase his utility) instead of truth-telling. Thus, each player $i$ answer $r_{i}=1$, "efficient allocation" will be $x^{*} \approx(38.3,38.3,38.3)$, but total utility with real players' types will be less than optimal since $x^{*} \neq x^{e f f}$.

So we have the incentive incompatibility problem: we can't just ask players about their types. There exists a range of mechanisms to solve the resource allocation problem with incentive incompatibility. Generally a mechanism $\rho=\langle S, x, t>$ consists of a set $S=\times_{i \in N} S_{i}$ of admissible bids of all players, a mapping $x(\cdot): S \rightarrow A$ for getting resource allocation from bids and a mapping $t(\cdot): S \rightarrow \mathbb{R}^{n}$ - some procedure for transfer utilities of players. A mechanism $\rho$ induces a strategic form game $\Gamma(\rho)=<N, S, \phi^{u, \rho}>$, where $\phi_{i}^{u, \rho}(s)=u_{i}(x(s))-t_{i}(s), i \in N$.

\subsection{Game process model and experimental data}

The Game process is implemented in the form of an iterative process as follows. At each iteration (step), $n$ bids (one bid from each player) are acquired and processed according to the rules of the Groves-Ledyard resource allocation mechanism (see appendix 1). The result of its operation is reported to all players. At the next step, any player may vary his bid, possibly affecting the result. The game process stops if none of the players vary his bid ("stop rule"), or the process reaches a maximum admissible step $T$ known to all players. Parameter $T$ was different in some game sessions: 60,20 , or 15 . The payoff of each player is defined as the profit at the last step.

So the last step is crucial because players receive payoffs on the last step. At the same time, the "stop rule" allows players to reach an agreement.

The participants of the experiments were Russian students of several State Universities of Moscow, Perm, Samara cities from faculties of Technology or Economics. Participants of one session studied the game rules and play in learning games, and then they were randomly allocated in groups of three and play final (test) games. We treat the results of each group as one separate game.

Notations: at each step $t$ players make bids $s_{1}(t), s_{2}(t), s_{3}(t)$ - bids of the first, second and third player accordingly. The situation at step $t$ is the tuple $s(t)=$ $\left(s_{1}(t), s_{2}(t), s_{3}(t)\right)$. Then, in accordance with the GL mechanism, they receive resources $x(s(t))$, give transfers $\tau(s(t))$ and their profits are $\phi(s(t))=u(x(s(t))-$ $\tau(s(t))$.

As experiments' results, we have data of 10 games, 10 sets of start-to-end situations $\left\{\left(s_{1}(1), s_{2}(1), s_{3}(1)\right), \ldots,\left(s_{1}\left(t_{g}^{e}\right), s_{2}\left(t_{g}^{e}\right), s_{3}\left(t_{g}^{e}\right)\right)\right\}$, where $t_{g}^{e}$ is the end step of game $g \in\{1, \ldots, 10\}$. Of course, in addition to the situations, we also have derived data: given resources, transfers, and profits in accordance with the GL mechanism. 


\section{The main approach: Nash bargaining solution}

We can treat the game process as a negotiation process among players: they bargain their profits. If they have reached a satisfactory result, they do not have a desire to change anything and therefore, will not change bids, and the game will stop by the "stop rule".

Initial Zeuthen-Hicks bargaining model considers the sequential interaction between two parties - the seller and the buyer (Harsanyi, 1956), (Vetschera, 2018). It was showed, that parties have an incentive to increase the value of the Nash bargaining objective function

$$
U(x)=\left(u_{i}(x)-u_{i}\left(d_{i}\right)\right) \cdot\left(u_{-i}(x)-u_{-i}\left(d_{-i}\right)\right)
$$

the maximum of which is the Nash Bargaining Solution (NBS) [Nash, 1950].

In case of three players it is not possible to treat negotiation as Zeuthen-Hicks Bargaining, but we can look at the Nash Bargaining objective function:

$$
U^{N a s h}(s, d)=\prod_{i=1}^{3}\left(\phi_{i}(s)-u_{i}\left(d_{i}\right)\right),
$$

where $d=(0,0,0)$ with $u(d)=(1,3,5)$, i.e. disagreement utility is base utility without transfers $p_{i}=0$ and resources $x_{i}=0$.

Due to GL mechanism, we use a profit $\phi$ of players instead of their utilities. Value of $U^{\text {Nash }}(s, d)$ in a concrete situation we will call "NBS value" for simplicity.

Another way to look at bargaining goal is "local NBS" version of the NBS:

$$
U_{l o c}^{N a s h}(s, t)=\prod_{i=1}^{3}\left(\phi_{i}(s)-\phi_{i}(s(t-1)) .\right.
$$

In our concrete case, $U^{N a s h}(s, d)$ and moreover $U_{l o c}^{N a s h}(s, t)$ can be positive if two of three multipliers in (8) and (9) are negative. Therefore, we use "shifted" objective functions:

$$
\begin{aligned}
U(s, d) & =\min _{i} \operatorname{sign}\left(\phi_{i}(s)-u_{i}\left(d_{i}\right)\right) \cdot\left|U^{N a s h}(s, d)\right| \\
U_{l o c}(s, t) & =\min _{i} \operatorname{sign}\left(\phi_{i}(s)-\phi_{i}(s(t-1))\right) \cdot\left|U_{l o c}^{N a s h}(s, t)\right| .
\end{aligned}
$$

The (10) and (11) functions are positive only if all multipliers (8) and (9) are positive. Additionally, for local NBS it means that player's profits have increased at step $t$.

Now let us proceed to the description of our approach to behavior analysis, incl.

\section{Behavior models}

We estimate a behavior model by share of players' decisions corresponding to the model and share of situations when some or all players made decisions corresponding to the model.

Let's denote $c$ - the count of all individual bids in our data, $C$ - the count of all situations. Note $c=3 C$ for our 3 -player games considered. Then the share of players decisions for some behavior $B$ is $\#\left\{s_{i}(t) \mid s_{i}(t) \in B\right\} / c$ and the share of situations 
for the behavior $B$ where at least $p$ players made decisions according to the $B$ is $\#\left\{s(t) \mid \#\left\{s_{i}(t) \in B \mid i=1,2,3\right\} \geq p\right\} / C$. (' $\# S^{\prime}$ means the number of elements in the set $S$ )

Now let us proceed to the description of the considered behavior models.

\section{Nash bargaining behavior}

- Firstly, we consider decisions that go into the direction of NBS increase, independently of whether the move is sufficiently large. We treat $s_{i}(t)$ to be Nashincreasing (NI) decision if:

$$
U\left(s^{i}(t), d\right)>U(s(t-1), d), i \in N,
$$

where $s^{i}(t)=\left(s_{i}(t), s_{-i}(t-1)\right)$ and $d=(0,0,0)$ :

- Further, we can define Real Nash increasing (Real NI) situations. We treat $s(t)$ to be Real Nash-increasing situation if it did increase the NBS value at step $t$ :

$$
U(s(t), d)>U(s(t-1), d) .
$$

- Similarly, instead of the NBS we can use local NBS. We treat $s_{i}(t)$ to be local Nash-increasing (LNI) decision if:

$$
U_{l o c}\left(s^{i}(t), t\right)>0,
$$

- and we treat $s(t)$ to be Real local Nash-increasing (Real LNI) situation if:

$$
U_{l o c}(s(t), t)>0 .
$$

Rational behavior All rational behavior models are based on the best response (BR) of a player to a situation on the previous step:

$$
b r_{i}(s(t-1))=\underset{y \in \mathbb{R}^{+}}{\arg \max } \phi_{i}\left(y_{i}, s_{-i}(t-1)\right) .
$$

Let's consider two rational behavior models.

- We treat $s_{i}(t)$ to be near best response decision with accuracy $\varepsilon(\operatorname{BR}(\varepsilon))$ if:

$$
\left|s_{i}(t)-b r_{i}(s(t-1))\right|<\varepsilon
$$

- We treat $s_{i}(t)$ to be Toward BR decision (TBR) if:

$$
\left\{\begin{array}{l}
s_{i}(t)=s_{i}(t-1), \text { if } b r_{i}(s(t-1))=s_{i}(t-1), \\
\left(s_{i}(t)-s_{i}(t-1)\right) /\left(b r_{i}(s(t-1))-s_{i}(t-1)\right)>0, \text { otherwise. }
\end{array}\right.
$$

Constant behavior Earlier we denoted bid of player $i$ at step $t$ as $s_{i}(t)$. Here we will denote a sequence of bids from step $t_{1}$ to step $t_{2}$ of player $i$ as $s_{i}\left(t_{1}, t_{2}\right)=$ $\left(s_{i}\left(t_{1}\right), s_{i}\left(t_{1}+1\right), \ldots, s_{i}\left(t_{2}\right)\right)$.

Some sequence of bids of one player $i$ from step $t_{s}$ to step $t_{e}>t_{s}$ with accuracy $\varepsilon$ is called Constant Behavior $C B\left(i, t_{s}, t_{e}, \varepsilon\right)$ if:

1. $\left|s_{i}\left(t_{s}, t_{e}\right)\right| \leq \varepsilon$

2. $\forall a<t_{s}, b>t_{e}:\left|s_{i}\left(a, t_{e}\right)\right|>\varepsilon \wedge\left|s_{i}\left(t_{s}, b\right)\right|>\varepsilon$ 
3. Not exists another $C B\left(i, t_{s}^{\prime}, t_{e}^{\prime}, \varepsilon\right)$ such that $t_{s}^{\prime}<t_{s}$ and $t_{s}<t_{e}^{\prime}<t_{e}$.

The above items describe mathematically results of the "algorithm" of sequential search for unchanged bids (with some accuracy $\varepsilon$ ): starting from the first step, we are looking for the bids sequence of players whose maximum and minimum differ by no more than $\varepsilon$.

The set of all $\mathrm{CB}$ with accuracy $\varepsilon$ is denoted as $C B(\varepsilon)$.

- We treat $s_{i}(t)$ to be Agree $C B$ decision (ACB) if the player $i$ doesn't change his decision at all - signal that he is agree with current allocation:

$$
s_{i}(t)=s_{i}(t-1)
$$

- We treat $s_{i}(t)$ to be Waiting $C B$ decision (WCB) if the player $i$ slightly changes his decision in order not to stop negotiation process:

$$
W C B(\varepsilon)=C B(\varepsilon) \backslash A C B
$$

- We treat $s_{i}(t)$ to be Rational $W C B$ decision (RWCB) if the player $i$ perform WCB toward his BR:

$$
R W C B(\varepsilon)=W C B(\varepsilon) \cap T B R
$$

Next section devoted to analysis based on the shares of the behavior models.

\section{Results}

\subsection{Individual decisions in YH Games}

In the table below (see figure 1), shares of individual decisions for the behavior models are presented. In the columns of the table results about all games, not consensus and consensus-ended games are depicted. We have equal number of not consensus and consensus-ended games, with similar amount of situations and individual decisions. Rows with couple behavior models, separated by 'Y' (for example - LNI $\backslash$ NI), correspond to decisions that suit a first behavior model (LNI) but not to a second one (NI).

The most observed models in all games are Local NI, Local NI $\backslash$ WCB(1), Local NI $\backslash$ NI and NI. It seems that non-Agree CBs occur significantly rare.

The data of games with consensus is similar to non-consensus games, but there is an exceptions:

1. Local Nash increasing models are found more often,

2. Slightly more Toward BR decisions,

3. Slightly more ACB decisions and slightly less $\mathrm{WCB}(1) \backslash \mathrm{ACB}$ decisions at once.

Generally most observed models in consensus and non-consensus games are almost equivalent. In consensus-ended games, players exhibit more intention to act accordingly to local NBS, i.e., to increase utility of all players. This result is similar to Local NI in games with YH mechanism [Korgin2020] but less evident: with YH we had simultaneously too less $\mathrm{WCB}(1)$ decisions and twice more (in \%) LNI decisions in consensus games.

Items 2 and 3 are negligible.

It is interesting that more $50 \%$ (199) of NI decisions are LNI (for LNI this is about $40 \%$ ). 


\begin{tabular}{|c|c|c|c|c|c|c|}
\hline & \multicolumn{2}{|r|}{ All } & \multicolumn{2}{|c|}{ NotConsensus } & \multicolumn{2}{|c|}{ Consensus } \\
\hline & num & $\%$ & num & $\%$ & num & $\%$ \\
\hline games & 10 & & 5 & & 5 & \\
\hline total iterations & 256 & & 135 & & 121 & \\
\hline \multirow[t]{2}{*}{ total decisions } & 768 & $100 \%$ & 405 & $100 \%$ & 363 & $100 \%$ \\
\hline & \multicolumn{6}{|c|}{ Rational behavior } \\
\hline $\mathrm{BR}(1)$ & 2 & $0 \%$ & 2 & $0 \%$ & 0 & $0 \%$ \\
\hline \multirow[t]{2}{*}{ Toward BR } & 176 & $23 \%$ & 86 & $21 \%$ & 90 & $25 \%$ \\
\hline & \multicolumn{6}{|c|}{ Constant behavior } \\
\hline Agree CB & 173 & $23 \%$ & 89 & $22 \%$ & 84 & $23 \%$ \\
\hline Waiting $C B(1) \backslash A C B$ & 55 & $7 \%$ & 34 & $8 \%$ & 21 & $6 \%$ \\
\hline \multirow[t]{2}{*}{ Rational WCB(1)\ACB } & 4 & $1 \%$ & 1 & $0 \%$ & $3 \mid$ & $1 \%$ \\
\hline & \multicolumn{6}{|c|}{ Nash Bargaining Solution Behavior } \\
\hline NI & 285 & $37 \%$ & 154 & $38 \%$ & 131 & $36 \%$ \\
\hline NI\WCB(1) & 260 & $34 \%$ & 136 & $34 \%$ & 124 & $34 \%$ \\
\hline Local NI & 524 & $68 \%$ & 252 & $62 \%$ & 272 & $75 \%$ \\
\hline LNI \WCB(1) & 354 & $46 \%$ & 175 & $43 \%$ & 179 & $49 \%$ \\
\hline LNI\NI & 325 & $42 \%$ & 152 & $38 \%$ & 173 & $48 \%$ \\
\hline$L N I \& L N>0$ & 19 & $2 \%$ & 6 & $1 \%$ & 13 & $4 \%$ \\
\hline
\end{tabular}

Fig. 1. Individual decisions in GL nonconsensus games and in consensus-ended GL games.

\subsection{Decision situations}

In the previous section, we see at bids of players and their intention toward BR, $\mathrm{CB}$ and NI, but view of negotiation process we can get from situations $s=\left(s_{1}, s_{2}, s_{3}\right)$ - behavior of player's group altogether. Again firstly we show general shares of situations. We consider two types of situations: when all players act according to the same behavior model and when at least one or two players act according to it.

\begin{tabular}{|c|c|c|c|c|c|c|}
\hline & \multirow{2}{*}{$\begin{array}{l}\text { All games } \\
\text { num }\end{array}$} & & \multicolumn{2}{|c|}{ Not Consensus } & \multicolumn{2}{|l|}{ Consensus } \\
\hline & & $\%$ & num & $\%$ & num & $\%$ \\
\hline games & 10 & & 5 & & 5 & \\
\hline \multirow[t]{2}{*}{ total situations } & 256 & $100 \%$ & 135 & $100 \%$ & 121 & $100 \%$ \\
\hline & \multicolumn{6}{|c|}{ Rational behavior } \\
\hline One BR(1) & 2 & $1 \%$ & 2 & $1 \%$ & 0 & $0 \%$ \\
\hline One TBR & 135 & $53 \%$ & 63 & $47 \%$ & 72 & $60 \%$ \\
\hline Two TBR & 37 & $14 \%$ & 20 & $15 \%$ & 17 & $14 \%$ \\
\hline \multirow[t]{2}{*}{ All TBR } & 4 & $2 \%$ & 3 & $2 \%$ & 1 & $1 \%$ \\
\hline & \multicolumn{6}{|c|}{ Constant behavior } \\
\hline One ACB & 127 & $50 \%$ & 69 & $51 \%$ & 58 & $48 \%$ \\
\hline Two ACB & 42 & $16 \%$ & 20 & $15 \%$ & 22 & $18 \%$ \\
\hline All ACB & 4 & $2 \%$ & 0 & $0 \%$ & 4 & $3 \%$ \\
\hline One Waiting $C B(1) \backslash A C B$ & 53 & $21 \%$ & 34 & $25 \%$ & 19 & $16 \%$ \\
\hline Two Waiting $C B(1) \backslash A C B$ & 2 & $1 \%$ & 0 & $0 \%$ & 2 & $2 \%$ \\
\hline All Waiting $C B(1) \backslash A C B$ & 0 & $0 \%$ & 0 & $0 \%$ & 0 & $0 \%$ \\
\hline One Rational WCB(1)\ACB & 4 & $2 \%$ & 1 & $1 \%$ & 3 & $2 \%$ \\
\hline
\end{tabular}

Fig. 2. Decision situations in GL games.

In figure 2 Rational and Constant behavior models are presented. We don't show "Two ..." and "All ..." for behaviors with negligible "One ..." situations. "All ..." situations have small shares at all, "Two ..." situations less than "One ..." and have no differences between nonconsensus and consensus games and "at least one ..." repeat individual decisions state. 
In figure 3 NBS models in situations are considered. "One..." models repeat decisions state. Situations with Real increase of non-local and local Nash function (Real LNI) has the second most observable case after "All Local NI" and they all are about half of situations!

Again share of behaviors decrease to 0 as we go from "One..." to "All..." situations. But we have exception with Local Nash function increasing behavior - it decrease from $75-90 \%$ down to $45-55 \%$. It split NI and LNI behavior - they have different template of "One..."-"All..." shares but have similar "Real ..." shares.

Concerning differences with consensus games for NI models, as with individual decisions, we see similar shares of situations. But for LNI models we see systematic shares growth: 9-15\% in consensus games. So when considering situations, the statement "players who demonstrate wish to increase local Nash function (i.e., utilities) came to an agreement" may take place. Alternatively, consensus-ended games have more players who demonstrate wish to increase utilities. Again in paper [Korgin2020] we had drastically more LNI-linked situations in consensus games (Real LNI was twice more).

Let's dive in Real NI and LNI data to try to reveal differences of NI and LNI models.

Figure 4 tells us that Real NI (Real LNI) situations toughly linked with NI (LNI) decisions in it. And Real NI (Real LNI) - LNI (NI) interlinks are not negligible.

Let's look at how Real NI and Real LNI interlinked with decisions. For that purpose we made two linear regression models for binary dependent variables 'Real NI' and 'Real LNI' from 10 binary independent variables 'One NI', Two NI', All NI','One LNI', 'Two LNI', All LNI','One TBR','Two TBR', 'One ACB','Two ACB'.

We found that the Real NI model has the mean squared error $\approx 0.15$ and the coefficient of determination $\approx 0.35$. Independent variables in regression coefficients decreasing order: 'All NI'( $\approx 1)$, 'Two NI' $(\approx 0.9)$, 'One $\mathrm{NI}^{\prime}(\approx 0.5)$, 'Two ACB'( $\approx$ $0.3)$. Coefficients of variables about LNI are negative and have small absolute values.

\begin{tabular}{|c|c|c|c|c|c|c|}
\hline & \multirow{2}{*}{$\begin{array}{l}\text { All games } \\
\text { num }\end{array}$} & \multirow[b]{2}{*}{$\%$} & \multicolumn{2}{|c|}{ Not Consensus } & \multicolumn{2}{|l|}{ Consensus } \\
\hline & & & num & $\%$ & num & $\%$ \\
\hline games & 10 & & 5 & & 5 & \\
\hline \multirow[t]{2}{*}{ total situations } & 256 & $100 \%$ & 135 & $100 \%$ & 121 & $100 \%$ \\
\hline & \multicolumn{6}{|c|}{ Nash increasing } \\
\hline One NI & 194 & $76 \%$ & 101 & $75 \%$ & 93 & $77 \%$ \\
\hline Two NI & 81 & $32 \%$ & 47 & $35 \%$ & 34 & $28 \%$ \\
\hline All NI & 10 & $4 \%$ & 6 & $4 \%$ & 4 & $3 \%$ \\
\hline One NI\WCB(1) & 183 & $71 \%$ & 92 & $68 \%$ & 91 & $75 \%$ \\
\hline Two NI\WCB(1) & 72 & $28 \%$ & 41 & $30 \%$ & 31 & $26 \%$ \\
\hline All NIIWCB(1) & 5 & $2 \%$ & 3 & $2 \%$ & 2 & $2 \%$ \\
\hline One Rational NI\WCB(1) & 75 & $29 \%$ & 41 & $30 \%$ & 34 & $28 \%$ \\
\hline Two Rational NI\WCB(1) & 13 & $5 \%$ & 8 & $6 \%$ & 5 & $4 \%$ \\
\hline All Rational NI\WCB(1) & 0 & $0 \%$ & 0 & $0 \%$ & 0 & $0 \%$ \\
\hline \multirow[t]{2}{*}{ Real NI } & 117 & $46 \%$ & 60 & $44 \%$ & 57 & $47 \%$ \\
\hline & \multicolumn{6}{|c|}{ Local Nash increasing } \\
\hline One Local NI & 208 & $81 \%$ & 100 & $74 \%$ & 108 & $89 \%$ \\
\hline Two Local NI & 190 & $74 \%$ & 92 & $68 \%$ & 98 & $81 \%$ \\
\hline All Local NI & 126 & $49 \%$ & 60 & $44 \%$ & 66 & $55 \%$ \\
\hline Real LNI & 121 & $47 \%$ & 58 & $43 \%$ & 63 & $52 \%$ \\
\hline
\end{tabular}

Fig. 3. Decision situations in GL games, NBS models. 
The second regression model about Real LNI has the mean squared error $\approx 0.1$ and the coefficient of determination $\approx 0.6$. Independent variables in regression coefficients decreasing order: 'All LNI' $\approx 0.85)$, 'Two LNI' $(\approx 0.15)$, 'All NI' $(\approx-0.15)$, 'Two NI' $\approx-0.1)$. Coefficients of variables about NI are negative.

An example of negotiation process for one game (game number 107) ended with consensus is presented in figure 5. At step 6 players show all behavior types. At step 7 individual decisions and situations are NBS agree and players 1 and 2 slightly change their bids. At step 8 only player 3 changes (slightly) his bid. May be players act cooperatively, and characteristics of situation at step 7 may be indicators of cooperative behavior. It is interesting that each player individually goes from some not CB model through WCB to ACB model.

\section{Conclusion}

The research conducted in (Korgin and Korepanov, 2020) states that indicators based on Nash bargaining value do allow us to predict possibilities for negotiation parties to reach a consensus in the unbalanced mechanism YH.

This research lead us to a negative result: with balanced mechanism GL we see that the Nash bargaining value cann't predict possibilities for negotiation parties to reach a consensus. We see only a small increase of LNI decisions in consensus-ended games. But we partially expect that since of balance property of the GL mechanism - it has no possibility to reduce total transfers. Nevertheless players have shown growth of "Local NI"-linked decisions and situations.

Since of negativity of the first result we look further at the Real NI and Real LNI behaviors. Some preliminar results about links between Real (L)NI and "One (L)NI" and from regression model about NBS value increasing prediction was obtained.

Perspectives of further research directions can be suggested. The first obvious direction is to make comparative analysis of several mechanisms on the data from

\begin{tabular}{|c|c|c|c|c|c|c|}
\hline & \multirow{2}{*}{$\begin{array}{l}\text { All games } \\
\text { num }\end{array}$} & & \multicolumn{2}{|c|}{ Not Consensus } & \multicolumn{2}{|l|}{ Consensus } \\
\hline & & $\%$ & num & $\%$ & num & $\%$ \\
\hline games & 10 & & 5 & & 5 & \\
\hline total situations & 256 & $100 \%$ & 135 & $100 \%$ & 121 & $100 \%$ \\
\hline Real NI & 117 & $46 \%$ & 60 & $44 \%$ & 57 & $47 \%$ \\
\hline Real NI \& no One NI & 1 & $0 \%$ & 1 & $1 \%$ & 0 & $0 \%$ \\
\hline Real NI \& no One LNI & 15 & $6 \%$ & 12 & $9 \%$ & 3 & $2 \%$ \\
\hline Real LNI & 121 & $47 \%$ & 58 & $43 \%$ & 63 & $52 \%$ \\
\hline Real LNI \& no One LNI & 0 & $0 \%$ & 0 & $0 \%$ & 0 & $0 \%$ \\
\hline Real LNI \& no One NI & 27 & $11 \%$ & 11 & $8 \%$ & 16 & $13 \%$ \\
\hline
\end{tabular}

Fig. 4. Decision situations in GL games, Real (Local)NI.

\begin{tabular}{|r|l|l|l|r|r|l|}
\hline Iteration & Player 1 & Player 2 & Player 3 & Real NI & Real LNI & \\
\hline 9 & ACB & ACB & ACB & 0 & 0 & game ended \\
\hline 8 & ACB & ACB & WCB\&LNI & 0 & 1 adjust? \\
\hline 7 & $\begin{array}{l}\text { WCB\&LNI\& } \\
\text { NI }\end{array}$ & WCB\&LNI & NI\&LNI & 1 & 1 cooperation? \\
\hline 6 & NI & LNI\&TBR & ACB & 1 & 0 \\
\hline
\end{tabular}

Fig. 5. Negotiation example. 
experimental games (Korgin and Korepanov, 2017) - to make equal sight to YH, GL and ADMM for example. All these mechanisms are from three different classes. And we can look not only at the end of negotiation process and the NBS behavior but on its causes - of course we can construct regression analysis of NBS value increasing more accurately: feature selection, treatment of categorical variables and confidence estimations. This is the second direction.

The third direction is connected with the experiment design - we have games when players take pay at each step of game - it may prevent they from negotiate and propagate different goal to maximize. So would it decrease the share of the NBS behavior is interesting question.

\section{Appendix}

\subsection{Groves-Ledyard mechanism}

The message of each agent at step 1 is the desired amount of the available resource $s_{i} \in[0, R]$. At the rest steps, the message of each agent is the desired resource allocation among all agents $s_{i}=\left(s_{i 1}, \ldots, s_{i n}\right), \sum_{j \in N} s_{i j} \in[0, R], s_{i} \in \mathbb{R}^{n}$.

The resource allotted to each agent is the mean of the messages of all agents about this agent, i.e.,

$$
x_{i}=\frac{1}{n} \sum_{j=1}^{n} s_{j i} .
$$

Each agent is assigned the additional transfer

$$
t_{i}=p_{i}-\frac{1}{n} \sum_{j=1}^{n} p_{j}
$$

where $p_{i}=\beta \sum_{j=1}^{n}\left(x_{j}-s_{i j}\right)^{2}$ gives penalty for "discrepancy" and $\beta=5 \times 10^{4}$ is the penalty strictness.

The resulting payoff of an agent is his utility from the resource gained at the last step of the game minus the assigned transfer: $\phi_{i}=u_{i}-t_{i}$.

\section{References}

Başar, T. and Maheswaran, R. T. (2003). Nash equilibrium and decentralized negotiation in auctioning divisible resources. Group Decision and Negotiation, 12(5), 361-395.

Boyd, S., Parikh, N., Chu, E. (2011). Distributed Optimization and Statistical Learning via the Alternating Direction Method of Multipliers. Foundations and Trends in Machine Learning, 3(1), 1-122.

Groves, T., Ledyard, J. O. (1977). Optimal allocation of public goods: A solution to the 'freerider' problem. Econometrica, 45, 783-809.

Groves, T., Ledyard, J. O. (1985). Incentive compatibility ten years later. Discussion Papers, 648.

Harsanyi, J. C. (1956). Approaches to the bargaining problem before and after the theory of games: A critical discussion of Zeuthen's, Hicks', and Nash's theories. Econometrica, 24(2), 144-157.

Korgin, N., Korepanov, V. (2016). An Efficient Solution of the Resource Allotment Problem with the Groves-Ledyard Mechanism under Transferable Utility. Automation and Remote Control, 77(5), 914-942. 
Korgin, N., Korepanov, V. (2017). Experimental gaming comparison of resource allocation rules in case of transferable utilities. International Game Theory Review, 19(2), 1750006.

Korgin, N., Korepanov, V. (2020). Nash bargaining solution as negotiation concept for resource allocation problem: analysis of experimental data. Contributions to Game Theory and Management, 13(0), 207-217.

Nash, J. F. (1950). The bargaining problem. Econometrica, 18(2), 155-162.

Yang, S., Hajek, B. (2005). Revenue and stability of a mechanism for efficient allocation of a divisible good, mimeo. Urbana Champaign: University of Illinois, 35 p.

Vetschera, R. (2018). Zeuthen-Hicks Bargaining in Electronic Negotiations. Group Decision and Negotiation, 1-20. 